\title{
Factores asociados al miedo y ansiedad en pacientes que asisten a consulta odontológica: Estudio Transversal
}

\section{Factors associated with fear and anxiety in patients attending a dental care: A cross-sectional study}

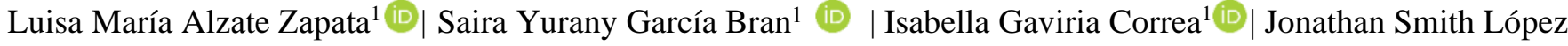 \\ Sarmiento $^{1}$ (D) | Juliana Ortiz Restrepo ${ }^{1}$ (D) John Querubín Franco Aguirre $^{1,2}$ (iD | Clara Liliana Gallego Gómez ${ }^{1}$ (i)
}

\section{f ACCESO ABIERTO}

Filiación Institucional

${ }^{1}$ Universidad Cooperativa de

Colombia, Facultad de Odontología,

Medellín. Colombia.

${ }^{2}$ Universidad de Antioquia,

Facultad de Odontología, Medellín, Colombia.

\section{Citación:}

Alzate-Zapata LM, García Bran SY, Gaviria-Correa I, López Sarmiento

JS, Ortíz-Restrepo J, Franco-Aguirre

JQ, Gallego-Gómez CL. Rev

Estomatol. 2021 Febrero

2021;29(1):e10929. DOI:

10.25100/re.v29i1.10929

Recibido: 13 Enero 2021

Evaluado: 18 Enero 2021

Aceptado: 22 Enero 2021

Publicado: 1 Marzo 2021

\section{Correspondencia:}

Clara Liliana Gallego Gómez.

Dirección Carrera 47 No. 37 Sur -

18, Medellín, Colombia, Teléfono 0544446065 ext 4112 Correo:

clara.gallego@ campusucc.edu.co

\section{Copyright:}

(C) Universidad del Valle.

\section{RESUMEN}

Antecedentes: Las personas que sienten miedo y ansiedad muestran peores condiciones de salud bucal, postergando las citas odontológicas hasta tal punto de evitarlas.

Objetivo: Determinar los factores asociados al miedo y la ansiedad en pacientes atendidos en las clínicas de la Facultad de Odontología de la Universidad Cooperativa de Colombia.

Materiales y métodos: Se realizó un estudio cuantitativo tipo descriptivo transversal con 198 pacientes atendidos en las clínicas de la facultad de Odontología. Se aplicaron las encuestas: Dental Fear Survey (DFS), Escala de Ansiedad Dental de Corah Modificada (MDAS) y una tercera encuesta para identificar: características sociodemográficas y elementos, situaciones y sensaciones asociados a miedo a la ansiedad durante la consulta.

Resultados: El 92,4\% de los participantes presentaron algún grado de miedo y el 48,7\% ansiedad en la consulta odontológica. La presencia del miedo y ansiedad se encontró asociada a experiencias previas en la consulta odontológica (Vp $<0,001)$, evidenciado en la mayor proporción de individuos con miedo alto y ansiedad severa cuya experiencia fue negativa en comparación con las frecuencias observadas para aquellos con clasificación de ansiedad leve o moderada y miedo nulo o bajo. Conclusiones: Las experiencias traumáticas previas están relacionadas con el miedo y la ansiedad dental y pueden influir en el adecuado desarrollo de la consulta odontológica, por lo que se recomienda indagar al paciente acerca de estas antes de iniciar atención odontológica.

PALABRAS CLAVE

Miedo; ansiedad; atención odontológica; escuela.

\section{ABSTRACT}

Background: People who feel fear and anxiety show worse oral health conditions than those who not, thus delaying dentist appointments to the point of cancelling all together.

Aim: Determine the degree of fear, anxiety and associated factors in patients seen in the clinics of the Faculty of Dentistry of the Universidad Cooperativa de Colombia.

Materials and methods: A quantitative cross-sectional descriptive study was conducted with patients treated in clinics of the Faculty of Dentistry, for a universe of 941 , a sample of 198 participants were calculated, not randomly selected. Three surveys were applied by the DFS (Dental Fear Survey), the MDAS (Anxiety Scale Modified Corah Dental) and a third survey to identify sociodemographic variables and factors associated with fear and anxiety.

Results: The $92.4 \%$ of the participants presented some degree of fear in the dental practice. $48.7 \%$ have anxiety to some degree. The presence of fear and anxiety was found associated with the evaluation of previous experiences in dental practice $(\mathrm{Vp}<0.001)$, evidenced in the highest proportion of individuals with high fear and anxiety whose experience was negative compared to the frequencies observed for those with mild or moderate anxiety and zero or low fear.

Conclusion: Previous traumatic experiences are related to fear and dental anxiety and can influence the proper development of the dental appointments, so it is recommended to inquire about these before starting with a dental treatment.

\section{KEY WORDS}

Fear; anxiety; dental care; school of dentistry. 


\section{Relevancia clínica}

El reconocimiento de las condiciones de salud del paciente, incluida la salud mental, es fundamental para el desarrollo de la práctica clínica en odontología.

\section{INTRODUCCIÓN}

La ansiedad se define como una serie de manifestaciones de tipo psíquico y mental que no se atribuyen a peligros reales y que se puede observar en forma de crisis o en un estado persistente, dichas manifestaciones se pueden desencadenar en episodios de pánico. ${ }^{1}$ El miedo es una sensación dada ante peligros reales, que se denomina también como una perturbación en la que se dan signos y síntomas similares a los de la ansiedad dentro de lo que se puede observar reacciones de tipo fisiológico y motor y pensamientos negativos. ${ }^{2}$ Según cifras de la Organización Mundial de la salud el número total de personas con trastornos de ansiedad en el mundo es de 264 millones y se ha ido presentando un aumento del $14.9 \%$ anualmente. En el continente americano el $7,7 \%$ de la población femenina sufre trastornos de ansiedad y el 3,6\% de los hombres. ${ }^{3}$ Dichas sensaciones se convierten en desadaptativas cuando se anticipan a un peligro irreal y de este modo se convierten en miedo y ansiedad clínica. ${ }^{4}$

Tanto el miedo como la ansiedad pueden interferir marcadamente en el desarrollo de las actividades de un individuo ya sea en el ámbito personal, laboral, social o familiar, al punto que este tipo de sensaciones le impiden desarrollarse de una determinada manera dentro de la sociedad. Se ha evidenciado que muchas personas han desarrollado miedo y ansiedad a la consulta odontológica lo que limita en gran medida la atención clínica. ${ }^{5}$

La ansiedad dental es una aprehensión más profunda que puede interferir en el tratamiento odontológico rutinario y debe ser tratada de una forma especial. A su vez el miedo al tratamiento odontológico se puede contemplar desde dos perspectivas, una como una reacción de fobia aprendida y condicionada por una experiencia negativa previa, y otra perspectiva que se puede dar como una respuesta de tipo psicológico ligada a temores que ya están presenten en el individuo. Ambas son de origen multifactorial, y puede ser por experiencias propias, por experiencias contadas por amigos o familiares, pero también puede ir asociada al trato que ha recibido por el odontólogo y a los errores que el paciente perciba en el tratamiento. ${ }^{6}$

Se ha demostrado que las personas que presentan miedo y ansiedad muestran peores condiciones de salud bucal, postergando las citas a la consulta odontológica hasta tal punto de evitarlas. La ansiedad dental ha sido clasificada en el quinto lugar de las situaciones más temidas por quienes acuden al servicio odontológico lo que trae como consecuencia evadir las citas odontológicas. ${ }^{7}$

A nivel mundial se ha reportado que la prevalencia del miedo y la ansiedad al tratamiento dental en un estudio sobre poblaciones encontró como Alemania presentaba niveles de miedo al dentista y ansiedad de un $8.6 \%$, Islandia de un $10 \%$, Australia de un $16.1 \%$, Singapur de $17.1 \%$, Holanda de un $24.3 \%$, China de $30 \%$ y Japón de $42.1 \% .^{1}$ En una investigación previa realizada en la Universidad Cooperativa de Colombia en el año 2011 se estudiaron los niveles de ansiedad en los pacientes que acudían a las clínicas de extracción dental de la facultad, encontraron niveles de ansiedad en $65.7 \%$ de los participantes. $^{8}$

Dentro los factores reportados en la literatura asociados a la presencia de miedo y ansiedad dental, se encontró un mayor nivel de ansiedad en edades entre los 40 a los 64 años, la mayor prevalencia de miedo fue encontrada en mujeres en cualquier edad, el estrato más bajo mostro mayores niveles de miedo dental que el alto. ${ }^{9}$

También, se ha reportado una mayor prevalencia de ansiedad dental ante experiencias previas pero no han abarcado otro tipo de factores asociados con miedo y ansiedad relacionados en con la consulta odontológica. El presente estudio tuvo como objetivo determinar los factores que causan miedo y ansiedad en pacientes atendidos en las clínicas de la Facultad de Odontología de la Universidad Cooperativa de Colombia.

\section{MATERIALES Y MÉTODOS}

\section{Diseño del estudio y cálculo de la muestra}

Se realizó un estudio descriptivo transversal en pacientes que fueron atendidos en las clínicas la facultad de Odontología de la Universidad Cooperativa de Colombia en la ciudad de Medellín (Colombia) durante el año 2019. Se realizó un cálculo de tamaño de muestra sobre una población total de 941 pacientes (histórico de atención en un semestre), intervalo de confianza del $95 \%$, precisión del $6 \%$ y una frecuencia esperada del $50 \%$ para miedo y ansiedad, obteniéndose una muestra de 208 individuos, los cuales fueron seleccionados de los pacientes que ingresaron a las clínicas de la Facultad durante el periodo de estudio.

\section{Criterios de selección}

Se incluyeron individuos mayores de 18 años de edad y se excluyeron aquellas personas que refirieron algún diagnós- 
tico de trastorno de ansiedad, analfabetas, individuos con deficiencias cognitivas o motoras o que realizaran alguna función asistencial en el área de la salud y quienes rechazaron su participación en el estudio. Se firmó el consentimiento.

\section{Evaluación del miedo, ansiedad y características sociodemográficas}

Se aplicaron tres encuestas a cada participante, las escalas Dental Fear Survey (DFS), la Escala de Ansiedad Dental de Corah Modificada (MDAS), y una encuesta para la obtención de la información sobre las características sociodemográficas y de los elementos, situaciones y sensaciones que generan intranquilidad durante la consulta odontológica, realizada para la investigación con base en estudios previos, y que fue validada previa prueba piloto.

La escala DFS de aplicación en adultos está establecida para identificar estímulos y reacciones específicas del miedo dental, es calificada a partir de la sumatoria de las respuestas en 20 ítems, cada uno con cinco opciones de respuesta en la escala Likert, las puntuaciones varían en un rango de 20 a 100 y se clasifican de acuerdo a los siguientes criterios: igual a 20 "sin miedo", entre 21 y 40 "miedo bajo", 41-79 "miedo moderado" y mayor o igual a 80 indicaría "miedo alto". Esta escala fue creada y validada por Kleinknecht y cols en $1984 ;{ }^{10}$ Coolidge y cols validaron la versión en español con resultados aceptables de sus propiedades psicométricas ${ }^{11} \mathrm{y}$ en Colombia ha sido aplicada por Vergara y cols en 460 individuos atendidos en consulta odontológica en la ciudad de Cartagena. ${ }^{12}$

La escala MDAS, validada por Humprhis y cols. ${ }^{13}$ para mejorar las propiedades sicométricas de la escala original de ansiedad dental (DAS), consta de 5 ítems, cada uno con 5 opciones de respuesta en la escala Likert, su puntuación total varia en un rango de 5 (ansiedad nula) a 25 (grado más alto de ansiedad), ha sido validada en el idioma español por Coolidge y cols. ${ }^{11}$ con resultados aceptables de sus propiedades psicométricas y aplicada en Colombia por Caicedo y cols en 913 pacientes odontológicos de la ciudad de Bogotá. ${ }^{14}$

\section{Análisis estadístico}

Para el análisis de la se realizó un análisis univariado de la información para la descripción de: las características sociodemográficas, las variables relacionadas a la consulta odontológica y el estado de miedo y ansiedad mediante la estimación de frecuencias relativas o medidas de resumen (tendencia central, dispersión y posición) según la naturaleza de la variable. Para explorar la asociación entre las características sociodemográficas y el miedo y la ansiedad se realizaron análisis bivariados a través de pruebas de correlación de Spearman, U de Mann Whitney o Kruskal Wallis con comparaciones múltiples según fuera el caso y con verificación previa del cumplimiento del supuesto de normalidad mediante el estadístico de Smirnov- Kolmogorov con corrección de Lilliefors. La asociación entre aspectos propios de la consulta odontológica con la clasificación de miedo y ansiedad se realizó a través de la prueba Chi cuadrado de Pearson y comparaciones múltiples de proporciones de columna con corrección de Bonferroni.

El análisis multivariado se aplicó para evaluar el efecto simultáneo de los factores asociados que permitiesen explicar los puntajes de miedo y ansiedad mediante un análisis de regresión lineal múltiple previa verificación del cumplimiento de los supuestos de: aleatoriedad de la variable dependiente, linealidad, incorrelacciòn de los residuos, no colinealidad, distribución normal y homocedasticidad de los residuos. La presentación de la información se realizó a través de tablas y gráficos de barras de error. En todos los análisis se tomó un valor $\mathrm{P}$ de significación estadística menor a 0,05 realizados a través del software IBM ${ }^{\circledR}$ SPSS $25 . \quad$ El presente investigación se considera de riesgo bajo según la resolución 8430 de 1993 y contó con el aval por el Comité Ético de la Universidad Cooperativa de Colombia, mediante el concepto bioético No 022-2019.

\section{RESULTADOS}

Los pacientes fueron reclutados durante dos semestres, entre los paciente que acudían a la atención odontológica en las clínicas de la Facultad. De una muestra estimada de 208 personas, se excluyeron 11 por la aplicación de los criterios descritos. El promedio de edad de los participantes fue de $49 \pm 15$ años, $69.0 \%$ eran mujeres, 63.9\% refirió tener un nivel de escolaridad básico de primaria o secundaria, en relación al estrato socioeconómico se evidenció una mayor frecuencia de individuos en los estratos 3 y 4 (65\%) (Tabla 1).

Entre los elementos que generan intranquilidad durante la consulta odontológica, se encontró que las agujas solas o en combinación con otros elementos (fresa, bisturí, materiales aplicados) fueron el objeto que con mayor frecuencia refirieron los participantes (62.4\%), en tanto que el $14.2 \%$ de las personas manifestaron que ningún elemento les generaba intranquilidad. La situación que genera mayor preocupación expresada por los pacientes, fue aquella en la que el estudiante u odontólogo pudiese equivocarse (37.6\%), en contraste un 33.5\% manifestó que ninguna de las situaciones expuestas le generaba intranqui- 
Alzate-Zapata y cols.

Tabla 1. Características sociodemográficas de la población de estudio.

\begin{tabular}{llc|c}
\hline & & Recuento & \% \\
\hline Sexo & Masculino & 61 & 31 \\
& Femenino & 136 & 69 \\
\hline Estrato & 1 & 9 & 4,6 \\
socioeconómico & 2 & 56 & 28,4 \\
& 3 & 103 & 52,3 \\
& 4 & 25 & 12,7 \\
& 5 & 2 & 1 \\
& 6 & 2 & 1 \\
\hline Nivel de & Primaria & 58 & 29,4 \\
escolaridad & Secundaria & 68 & 34,5 \\
& Técnico & 28 & 14,2 \\
& Tecnológico & 14 & 7,1 \\
\cline { 2 - 4 } & Universitario & 28 & 14,2 \\
& Posgrado & 1 & 0,5 \\
\hline & & Media \pm DE & Mín- Máx \\
\cline { 2 - 4 } & Edad (años) & $49 \pm 15$ & $18-86$ \\
\hline
\end{tabular}

DE: desviación estándar; Min: mínimo; Max: máximo.

Tabla 2. Frecuencia de miedo y ansiedad.

\begin{tabular}{llc|c}
\hline & & Recuento & \% \\
\hline Clasificación de & Sin miedo & 15 & $7,6 \%$ \\
miedo & Miedo bajo & 100 & $50,8 \%$ \\
& Miedo moderado & 67 & $34,0 \%$ \\
& Miedo alto & 15 & $7,6 \%$ \\
\hline Clasificación de & Leve o nula & 101 & $51,3 \%$ \\
ansiedad & Moderada & 56 & $28,4 \%$ \\
& Elevada & 11 & $5,6 \%$ \\
& Severa & 29 & $14,7 \%$ \\
\hline
\end{tabular}

lidad. Con relación a las sensaciones expuestas, el dolor solo o en combinación con otras condiciones (sangrado, nauseas, sensibilidad, presión) fue el evento que en mayor proporción refirieron los individuos del estudio (65.9\%), solo un $7.6 \%$ de las personas manifestaron que ninguna de las sensaciones le generaba intranquilidad.

La evaluación del miedo en la población de estudio, evidenció que el $92.4 \%$ de los participantes presentaron algún estado de esta condición durante la consulta odontológica, en relación a la ansiedad se evidenció una frecuencia del $48.7 \%$ de personas que presentaron ansiedad en grado moderado, elevado o severo (Tabla 2).

La clasificación de miedo y ansiedad se encontró asociada con la evaluación de las experiencias previas en la consulta odontológica ( $\mathrm{Vp}$ Chi cuadrado $<0,001$ ), evidenciado puntualmente en la mayor proporción de individuos con miedo alto $(66.7 \%) \quad y$ ansiedad severa $(48.3 \%)$ cuya experiencia fue negativa en comparación con las frecuencias observadas de experiencias negativas para aquellos con clasificación de ansiedad leve o moderada y miedo nulo o bajo (Figuras 1y 2).

Se encontró una correlación leve e inversa entre los puntajes de miedo (rho spearman $=-0.247$ ) y ansiedad (rho spearman $=-0.282$ ) con la edad, en tal sentido, se observó que una alta proporción de personas con las mayores edades presentaron bajos puntajes en las escalas, categorizados en los grados de ansiedad leve o nula y bajo miedo. No se encontró asociación entre el miedo y ansiedad con el sexo, sin embargo, se observó una tendencia hacia mayores puntajes en la escala de ansiedad para las mujeres ( $\mathrm{Vp} \mathrm{U}$ de Mann Whitney $=0.05$ ) (Tabla 3).

El estrato socioeconómico, se encontró como un factor asociado a la ansiedad (Vp kruskal Wallis = 0.027), puntualmente en la comparación de los puntajes entre los individuos de estratos 1 y 4 (Vp U de Mann Whitney con corrección de Bonferroni $=0.020)$, los cuales fueron menores en este último grupo (Tabla 3). Referir intranquilidad frente alguna sensación, situación o elemento durante la consulta odontológica, se encontró asociado al miedo y la ansiedad dental (Vp U de Mann Whitney $\leq 0,001)$, es así como se observaron mayores puntajes en ambas escalas para aquellos individuos que reportaron intranquilidad para alguno de los factores mencionados (Tabla 4).

El modelo de regresión utilizado evidenció la asociación confundida de la edad y la intranquilidad a sensaciones durante la consulta (no aplicadas del modelo) con el miedo y la ansiedad, a su vez demostró el efecto simultáneo de la intranquilidad a elementos y situaciones y de las experiencias previas en consulta odontológica sobre ambas condiciones, explicando respectivamente el $31.2 \%$ y $32.5 \%$ de la variación en los puntajes de ansiedad y miedo. 


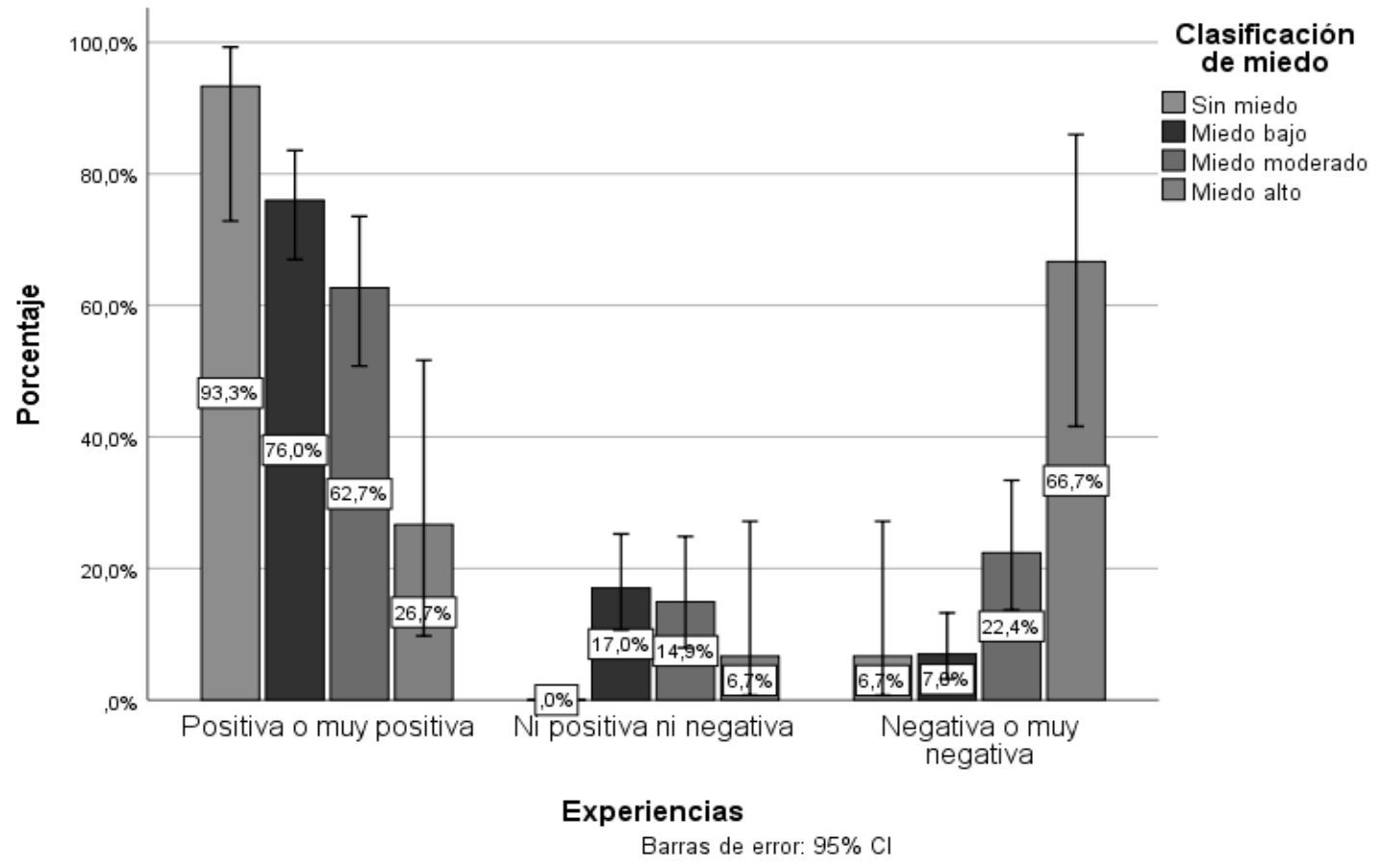

Figura 1. Clasificación del miedo según experiencias previas durante la consulta odontológica.

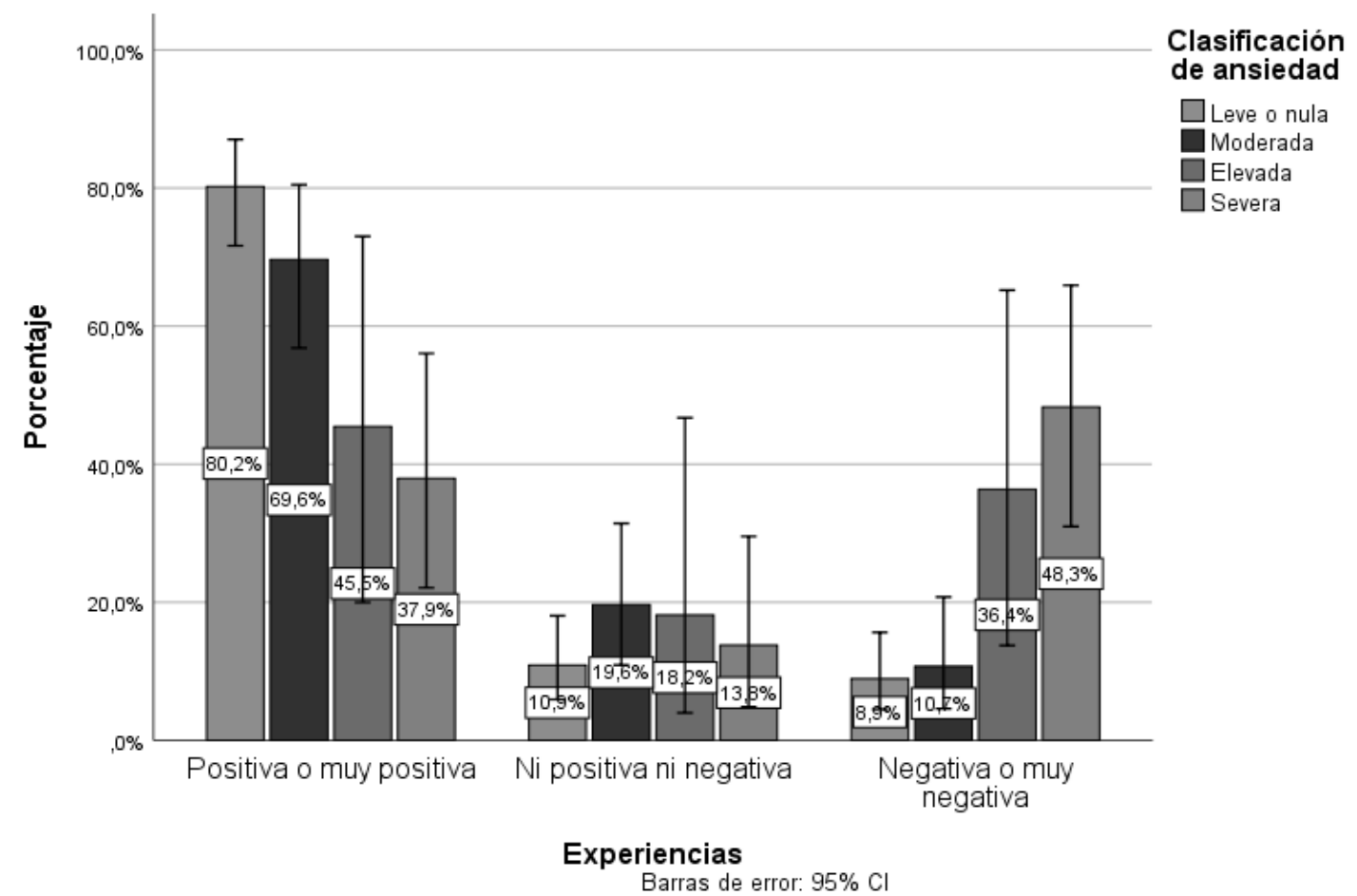

Figura 2. Clasificación de la ansiedad según experiencias previas durante la consulta odontológica. 
Alzate-Zapata y cols.

Tabla 3. Puntaje de miedo y ansiedad según características sociodemográficas.

\begin{tabular}{|c|c|c|c|c|c|c|c|}
\hline & & \multicolumn{3}{|c|}{ Puntaje miedo } & \multicolumn{3}{|c|}{ Puntaje ansiedad } \\
\hline & & Mediana (RI) & Mín.- máx. & Valor $\mathbf{P}$ & Mediana(RI) & Mín.- máx & Valor $\mathbf{P}$ \\
\hline \multirow[t]{2}{*}{ Sexo } & Masculino & $35(26-40)$ & $20-82$ & \multirow{2}{*}{$0,287^{*}$} & $8(5-11)$ & $5-17$ & \multirow[t]{2}{*}{$0,05^{*}$} \\
\hline & Femenino & $36(28-58)$ & $20-94$ & & $9(6-12)$ & $5-25$ & \\
\hline \multirow{6}{*}{$\begin{array}{l}\text { Estrato } \\
\text { socioeconómico }\end{array}$} & 1 & $54(34-54)$ & $24-93$ & \multirow{6}{*}{$0,275^{* *}$} & $12(9-19)^{* * *}$ & $6-23$ & \multirow{6}{*}{$0,027 * *$} \\
\hline & 2 & $37(28-54)$ & $20-86$ & & $10(6-11)$ & $5-23$ & \\
\hline & 3 & $36(27-56)$ & $20-94$ & & $8(6-12)$ & $5-25$ & \\
\hline & 4 & $35(26-41)$ & $20-85$ & & $7(5-9)^{* * *}$ & $5-16$ & \\
\hline & 5 & $45(44-45)$ & $44-45$ & & $8(5-11)$ & $5-11$ & \\
\hline & 6 & $25(23-27)$ & $23-27$ & & $6(6-6)$ & $6-6$ & \\
\hline \multirow{6}{*}{$\begin{array}{l}\text { Nivel de } \\
\text { escolaridad }\end{array}$} & Primaria & $33(23-54)$ & $20-94$ & \multirow{6}{*}{$0,194 * *$} & $8(6-11)$ & $5-25$ & \multirow{6}{*}{$0,574 * *$} \\
\hline & Secundaria & $35(28-53)$ & $20-86$ & & $8(6-11)$ & $5-21$ & \\
\hline & Técnico & $38(31-55)$ & $20-89$ & & $9(7-11)$ & $5-19$ & \\
\hline & Tecnológico & $34(23-39)$ & $20-63$ & & $7(6-10)$ & $5-10$ & \\
\hline & $\begin{array}{l}\text { Universitari } \\
\mathrm{o}\end{array}$ & $43(33-68)$ & $20-87$ & & $9(6-15)$ & $5-22$ & \\
\hline & Posgrado & $49(49-49)$ & $49-49$ & & $11(11-11)$ & $11-11$ & \\
\hline
\end{tabular}

Tabla 4. Puntaje de miedo y ansiedad según intranquilidad a elementos, situaciones y sensaciones en consulta odontológica.

\begin{tabular}{|c|c|c|c|c|c|c|c|}
\hline & & \multicolumn{3}{|c|}{ Puntaje miedo } & \multicolumn{2}{|c|}{ Puntaje ansiedad } & \multirow[b]{2}{*}{ Valor P* } \\
\hline & & $\begin{array}{l}\text { Mediana } \\
\quad(\text { RI) }\end{array}$ & Mín- Max & Valor $\mathbf{P}^{*}$ & $\begin{array}{c}\text { Mediana } \\
\text { (RI) }\end{array}$ & Mín- Max & \\
\hline \multirow{2}{*}{$\begin{array}{l}\text { Intranquilidad a algún } \\
\text { elemento }\end{array}$} & No & $22(20-24)$ & $20-43$ & \multirow[t]{2}{*}{$<0,001$} & $5(5-5)$ & $5-6$ & \multirow[t]{2}{*}{$<0,001$} \\
\hline & $\mathrm{Si}$ & $38(31-56)$ & $20-94$ & & $9(7-12)$ & $5-25$ & \\
\hline \multirow{2}{*}{$\begin{array}{l}\text { Intranquilidad a } \\
\text { situaciones }\end{array}$} & No & $29(23-36)$ & $20-74$ & \multirow[t]{2}{*}{$<0,001$} & $6(5-9)$ & $5-17$ & \multirow[t]{2}{*}{$<0,001$} \\
\hline & $\mathrm{Si}$ & $41(32-60)$ & $20-94$ & & $9(7-13)$ & $5-25$ & \\
\hline \multirow{2}{*}{$\begin{array}{l}\text { Intranquilidad frente a } \\
\text { alguna sensación }\end{array}$} & No & $21(20-24)$ & $20-83$ & \multirow[t]{2}{*}{$<0,001$} & $5(5-6)$ & $5-18$ & \multirow[t]{2}{*}{0,001} \\
\hline & $\mathrm{Si}$ & $37(29-54)$ & $20-94$ & & $9(6-11)$ & $5-25$ & \\
\hline
\end{tabular}


Alzate-Zapata y cols.

Tabla 6. Modelo de regresión: puntaje en la escala de ansiedad explicado por los factores asociados.

\begin{tabular}{lcccc}
\hline & $\begin{array}{c}\text { Coeficiente } \\
\text { B }\end{array}$ & $\begin{array}{c}\text { IC* 95 \% } \\
\text { para B }\end{array}$ & Valor P & $\begin{array}{c}\text { R cuadrado } \\
\text { ajustado }\end{array}$ \\
\hline $\begin{array}{l}\text { Experiencias en anteriores consultas } \\
\text { odontológicas (Positiva(1) / Ni positiva ni } \\
\text { negativa (2)/ Negativa (3) }\end{array}$ & 2,11 & $1,40-2,82$ & $<0,001$ \\
\hline $\begin{array}{l}\text { Intranquilidad a situaciones (No/Si) } \\
\text { Intranquilidad a algún elemento (No/Si) }\end{array}$ & 2,14 & & \\
\hline $\begin{array}{l}\text { Intranquilidad frente a alguna sensación } \\
\text { (No/Si) }\end{array}$ & $-0,59$ & $0,97-3,30$ & $<0,001$ & 0,312 \\
\hline Edad & $-0,31$ & $2,02-5,15$ & $<0,001$ \\
\hline
\end{tabular}

*IC $=$ Intervalo de confianza

Tabla 7. Modelo de regresión: puntaje en la escala de miedo explicado por los factores asociados.

\begin{tabular}{|c|c|c|c|c|}
\hline & $\begin{array}{l}\text { Coeficientes } \\
\text { B }\end{array}$ & $\begin{array}{c}\text { IC } * 95,0 \% \\
\text { para } B\end{array}$ & Valor $\mathbf{P}$ & $\begin{array}{c}R \text { cuadrado } \\
\text { ajustado }\end{array}$ \\
\hline $\begin{array}{l}\text { Experiencias en anteriores consultas odontológicas } \\
\text { Positiva(1) / Ni positiva ni negativa(2) / Negativa)(3) }\end{array}$ & 8,15 & $5,14-11,16$ & $<0,001$ & \multirow{5}{*}{0,325} \\
\hline Intranquilidad a situaciones $(\mathrm{No} / \mathrm{Si})$ & 11,36 & $6,45-16,27$ & $<0,001$ & \\
\hline Intranquilidad a algún elemento (No/Si) & 15,47 & $8,88-22,06$ & $<0,001$ & \\
\hline Intranquilidad frente a alguna sensación & 2,61 & $-6,65-11,88$ & 0,579 & \\
\hline Edad & $-0,092$ & $-0,25-0,07$ & 0,258 & \\
\hline
\end{tabular}

*IC = Intervalo de confianza

De esta forma, se encontró que quienes manifiesten intranquilidad frente alguna situación y elemento durante la consulta odontológica, aumentarían respectivamente en 2.13 y 3.6 puntos en la escala de ansiedad en relación a quienes lo niegan, de forma similar en la escala de miedo se aumentaría en 11.4 y 15.5 puntos; respecto a las experiencias previas, haberlas referido como negativas en cualquier grado representaría 16.3 puntos más en la escala de miedo y 4.2 más en la escala de ansiedad en comparación con aquellos que manifiestan una experiencia positiva o muy positiva (Tablas 6 y 7 ).

\section{DISCUSIÓN}

El miedo y la ansiedad dental son situaciones que se presentan diariamente en la consulta odontológica lo cual puede interferir en el tratamiento odontológico rutinario y debe ser tratada de una forma especial y puede ser conse- cuencia de experiencias negativas previas. Según los resultados obtenidos en esta investigación, de la población evaluada se encontró una alta prevalencia de miedo (92.4\%), y con relación a éste, la ansiedad presento porcentajes más bajos (48.7\%), resultados comparables con lo reportado por Arrieta y cols. (2013) quienes reportaron un alto porcentaje de ansiedad (62.8\%) y miedo dental (77.1\%); demostrando que es un fenómeno que se presentan con alta frecuencia en la consulta odontológica, lo que puede dificultar la atención del paciente y la realización de algún tratamiento. ${ }^{15}$

El presente estudio no encontró asociación entre el miedo y ansiedad con el sexo sin embargo, se evidenció una tendencia de mayores puntajes en la escala de ansiedad para las mujeres, confirmando los resultados reportados en el estudio de Caycedo y cols (2008), donde se observó que las mujeres presentaron una mayor prevalencia de ansiedad dental en relación a los hombres. ${ }^{16}$ 
Con respecto al estrato socioeconómico y el nivel de escolaridad se encontró que la mayoría de la población estudiada terminaron sus estudios secundarios y que el mayor porcentaje en estrato socioeconómico fue bajo; esto pude deberse a que a la facultad de la Universidad Cooperativa asisten participantes que debido a su estrato socioeconómico han dejado avanzar las alteraciones dentales y requieren tratamientos más invasivos, lo que puede ser comparable a lo reportado por Locker y Liddell, quienes no encontraron diferencias en cuanto al nivel de escolaridad ni al estrato socioeconómico. ${ }^{17}$

Previos estudios han reportado que, ante experiencias traumáticas previas, se observan altos porcentajes de miedo de un $89.7 \%$ y ansiedad en un $76 \%,{ }^{10,15}$ mientras que en la presente investigación se encontraron porcentajes de $66.7 \%$ para el miedo alto y un $48.3 \%$ para ansiedad severa. En ambos estudios se encontraron resultados más altos de miedo ya que cuando un individuo ha sido sometido a tratamientos complejos o invasivos puede presentar mayor dificultad para enfrentarse nuevamente a cualquier tipo de experiencia odontológica.

Al evaluar otros factores que podrían influir en la presencia de miedo y ansiedad ante el tratamiento odontológico, se observó que, el trato del personal de salud, no genera miedo y ansiedad en la población estudiada; por el contrario, el instrumental y las sensaciones (sangrado excesivo, dolor, náuseas, presión, otros) fueron factores desencadenantes y contraproducentes en el estado emocional de los participantes en el momento de realizar un tratamiento.

En el presente estudio se encontró como el miedo y la ansiedad se relacionan con factores como las experiencias traumáticas previas, el instrumental, siendo las agujas la de mayor porcentaje $(62.4 \%)$ y que el estudiante se equivoque con un porcentaje de $37.6 \%$ dentro de las diferentes situaciones, por último, la sensación que causa mayor intranquilidad es el dolor $(65.9 \%)$ durante los múltiples tipos de tratamientos odontológicos de las clínicas tenidas en cuenta en el estudio. Por tanto, es importante que el clínico reconozca la presencia de miedo y ansiedad en los pacientes, situaciones que tendrían serias implicaciones en los tratamientos propuestos y la adherencia de los pacientes a los mismos, por lo que se propone el uso de sus escalas de medición dentro del desarrollo de la historia clínica.

Una posible limitación para el estudio es la recolección de la información dentro del ambiente institucional, lo que puede en alguna medida minimizar el fenómeno de estudio.

Rev Estomatol. 2021;29(1):e10929

\section{CONCLUSIONES}

El miedo y la ansiedad dental son muy comunes en la consulta odontológica y pueden ser difíciles de percibir por el profesional tratante, por lo cual es aconsejable incluir dentro de la historia clínica odontológica las escalas que evalúen dichas sensaciones con el fin de lograr tener un mejor manejo y una adecuada atención en aquellos pacientes que las padecen.

Las experiencias traumáticas previas están relacionadas con el miedo y la ansiedad dental y pueden influir en el adecuado desarrollo de la consulta odontológica, por lo que se recomienda indagar al paciente acerca de estas previo a la atención odontológica y en caso de ser negativas buscar alternativas en conjunto para que éste se sienta más cómodo durante el tratamiento.

\section{AGRADECIMIENTOS}

Los autores agradecen a los participantes del estudio por su tiempo y dedicación, a las Directivas de la Facultad de Odontología por brindarnos la oportunidad para la realización de estudio y los Profesores de investigación quienes nos apoyaron con sus valiosos comentarios para el desarrollo del estudio. A Isabella Gaviria Correa, los frutos que dejaste en la tierra, hoy los celebras desde el cielo, que tu sonrisa siga brillando por cada uno de ellos, gracias por haber creído y trabajado con tanto amor en este proyecto.

\section{DECLARACIÓN DE CONFLICTO DE INTERÉS}

Los autores manifiestan no tener ningún conflicto de interés para el desarrollo del presente estudio

\section{FUENTES DE FINANCIACIÓN}

El presente proyecto fue financiado con recursos para el desarrollo de la investigación de la Universidad Cooperativa de Colombia.

\section{REREFENCIAS}

1. Centro de Formación Odontológico. Athenea Dental Institute. Introducción a la Odontología Interdisciplinar[ Internet]. España [Internet]. Centro de Formación Odontológico. 2016. Available from: https://atheneainstitute.com/master-ortointerdisciplinar/

2. Rojas Alcayaga G. Rios, M. Herrera A. Ansiedad dental : Evaluación y tratamiento. Av Odontoestomatol [Internet]. 2013;30:39-46. Available from: http://scielo.isciii.es/scielo.php?script=sci_arttext\&pid=S0213 $-12852014000100005$

3. Organización Mundial de la Salud OP de la salud. Depresión y otros trastornos mentales comunes. Estimaciones sanitarias mundiales. [Internet]. 2017. Available from: http://iris.paho.org/xmlui/bitstream/handle/123456789/34006/ PAHONMH17005-spa.pdf 
Alzate-Zapata y cols.

4. Ortega, J, Zubeidat, I, Sierra J. Ansiedad, angustia y estrés: tres conceptos a diferenciar. Rev Mal-estar E Subjetividade [Internet]. 2003;3(1). Available from: http://pepsic.bvsalud.org/scielo.php?script=sci_arttext\&pid=S 1518-61482003000100002

5. Lima M, Casanova Y. Miedo, ansiedad y fobia al tratamiento estomatológico. Humanidades Médicas [Internet]. 2006;6(1):21. Available from: http://scielo.sld.cu/scielo.php?script=sci_arttext\&pid=S172781202006000100007

6. Marquez, J, Navarro, M, Cruz, D, Gil J. ¿Por qué se le tiene miedo al dentista? RCOE [Internet]. 2004;9:165-74. Available from:

http://scielo.isciii.es/scielo.php?script=sci_arttext\&pid=S1138 $-123 X 2004000200003$

7. Hmud R, Lj W. Ansiedad dental: causas, complicaciones y métodos de manejo. Rev Mínima Interv En Odontol [Internet]. 2009;2(1):237-48. Available from: http://www.miseeq.com/s2-1-8.pdf

8. Vásquez C, Maya L, Córdoba J JS. Niveles de ansiedad prequirúrgicos en pacientes adultos Jóvenes atendidos en Clínica de Exodoncia de la UCC. 2011.p. 1.

9. Organización Mundial de la Salud C de P. OPS. La salud bucodental es esencial para la salud general[Internet]Estados Unidos. [Internet]. Oficina Regional para las Américas de la Organización Mundial de la Salud. 2003. Available from: https://www.paho.org/hq/index.php?option=com_content\&vie $\mathrm{w}=$ article $\& \mathrm{id}=8387: 2013$-oral-health-vital-overallhealth\&Itemid $=135 \&$ lang $=$ es

Kleinknecht RA, Thorndike RM, McGlynn FD, Harkavy J. Factor analysis of the dental fear survey with cross-validation. J Am Dent Assoc. 1984;108: 59-61.

11. Coolidge T, Chambers M, Garcia L, Heaton L, Coldwell S. Psychometric properties of Spanish-language adult dental fear measures. BMC Oral Health. 2008; 15(8) doi:10.1186/1472-6831-8-15

12. Arrieta K, Díaz S, Verbel J, Hawasly N. Factores asociados a sintomatología clínica de miedo y ansiedad en pacientes atendidos en Odontología. Rev Clin Med Fam.2013; 6(1): 17 24 http://dx.doi.org/10.4321/S1699-695X2013000100004.

13. Humphris GM, Morrison T, Lindsay SJE: The Modified Dental Anxiety Scale: Validation and United Kingdom norms. Community Dent Health. 1995, 12: 143-150.

14. Caycedo C, Cortes O, Gama R, Rodríguez H, Colorado P, Caycedo $\mathrm{M}$, et al. Ansiedad al tratamiento odontológico: características y diferencias de género. Rev Suma psicológica.2008; 15 (1): 259-278.

15. Arrieta, K, Díaz, S, Verbel , J, Nathalie Hawasly N. Factores asociados a sintomatología clínica de miedo y ansiedad en pacientes atendidos en Odontología. Rev Clínica Med Fam [Internet]. 2013;6(1):17-24. Available from: http://scielo.isciii.es/scielo.php?script=sci_arttext\&pid=S1699 $-695 X 2013000100004$

16. Caycedo C, Cortes O, Gama R; Rodríguez H, Colorado P; Caycedo $\mathrm{M}$, et al. Ansiedad al tratamiento odontológico: características y diferencias de género Suma psicológica.2008; 15(1): 259-278.

17. Locker D, Liddell A. Correlates of dental anxiety among older adults. J Dent. Res1991; 70(3):198-203.

Rev Estomatol. 2021;29(1):e10929 